\title{
A PHOTOMETRIC METALLICITY ESTIMATE OF THE VIRGO STELLAR OVERDENSITY
}

\author{
Deokkeun An ${ }^{1}$, Jennifer A. Johnson ${ }^{2}$, Timothy C. Beers ${ }^{3}$, Marc H. Pinsonneault ${ }^{2}$, Donald M. Terndrup ${ }^{2,4}$, \\ Franck Delahaye ${ }^{5,6,7}$, Young Sun LeE ${ }^{3}$, Thomas Masseron ${ }^{2}$, and Brian Yanny ${ }^{8}$ \\ ${ }^{1}$ Infrared Processing and Analysis Center, California Institute of Technology, Mail Stop 100-22, Pasadena, CA 91125, USA; deokkeun@ipac.caltech.edu \\ ${ }^{2}$ Department of Astronomy, Ohio State University, 140 West 18th Avenue, Columbus, OH 43210, USA \\ ${ }^{3}$ Department of Physics \& Astronomy and JINA (Joint Institute for Nuclear Astrophysics), Michigan State University, E. Lansing, MI 48824, USA \\ ${ }^{4}$ Division of Astronomical Sciences, National Science Foundation, 4201 Wilson Blvd., Arlington, VA 22230, USA \\ ${ }^{5}$ Service d'Astrophysique, CEA/DSM/IRFU/SAp, CEA Saclay, 91191 Gif-sur-Yvette Cedex, France \\ ${ }^{6}$ Centre Lasers Intenses et Applications (CELIA), 351 Cours de la Libération, 33405 Talence Cedex, France \\ ${ }^{7}$ LERMA, Observatoire de Paris, CNRS, Université Paris Diderot, 5 Place Jules Janssen, 92190 Meudon, France \\ ${ }^{8}$ Fermi National Accelerator Laboratory, P.O. Box 500, Batavia, IL 60510, USA \\ Received 2009 July 4; accepted 2009 November 5; published 2009 November 24
}

\begin{abstract}
We determine photometric metal abundance estimates for individual main-sequence stars in the Virgo Overdensity (VOD), which covers almost $1000 \mathrm{deg}^{2}$ on the sky, based on a calibration of the metallicity sensitivity of stellar isochrones in the $\mathrm{gri}$ filter passbands using field stars with well-determined spectroscopic metal abundances. Despite the low precision of the method for individual stars, we derive $[\mathrm{Fe} / \mathrm{H}]=-2.0 \pm 0.1$ (internal) \pm 0.5 (systematic) for the metal abundance of the VOD from photometric measurements of 0.7 million stars in the northern Galactic hemisphere with heliocentric distances from $\sim 10 \mathrm{kpc}$ to $\sim 20 \mathrm{kpc}$. The metallicity of the VOD is indistinguishable, within $\Delta[\mathrm{Fe} / \mathrm{H}] \leqslant 0.2$, from that of field halo stars covering the same distance range. This initial application suggests that the Sloan Digital Sky Survey gri passbands can be used to probe the properties of main-sequence stars beyond $\sim 10 \mathrm{kpc}$, complementing studies of nearby stars from more metallicity-sensitive color indices that involve the $u$ passband.
\end{abstract}

Key words: Galaxy: abundances - Galaxy: evolution - Galaxy: formation - Galaxy: halo - Galaxy: stellar content - Galaxy: structure

\section{INTRODUCTION}

The structure, chemistry, and kinematics of the stellar halo of the Galaxy, with its predominantly old and metal-poor populations, collectively preserve a detailed record of our Galaxy's formation in the early universe (e.g., Eggen et al. 1962; Searle \& Zinn 1978). Thanks to large-area surveys such as the Sloan Digital Sky Survey (SDSS; York et al. 2000; Stoughton et al. 2002; Abazajian et al. 2003, 2004, 2005, 2009; AdelmanMcCarthy et al. 2006, 2007, 2008), recent studies have revealed that the halo is marked by numerous stellar substructures. The presence of these lumpy and complex substructures (both in real and phase space) are in qualitative agreement with models for the formation of the stellar halo through the hierarchical merging and accretion of low-mass subhalos (e.g., Bullock \& Johnston 2005).

Among the various substructures discovered to date, the Virgo Overdensity (VOD) is one of the most striking. It was discovered as a stellar overdensity of main-sequence stars in SDSS; star counts in the region toward Virgo are enhanced by a factor of 2 above the background stellar distribution (Jurić et al. 2008). The overdensity covers almost $1000 \mathrm{deg}^{2}$, and it appears to span a wide range of heliocentric distances of $\sim 5-20 \mathrm{kpc}$. The overdensity seems to be associated with clumps of RR Lyrae stars (Vivas et al. 2001; Duffau et al. 2006) and turnoff stars (Newberg et al. 2002), but it is less likely to be connected with the leading tidal tail of the Sagittarius (Sgr) dwarf galaxy (Newberg et al. 2007).

At present, metallicity estimation from broadband photometry is the only practical means of obtaining metal abundances for a large number of faint objects such as those in the VOD. Such methods are based on the relative sensitivity of stellar colors to photospheric abundances over a wide wavelength baseline. The clear advantage of using a photometric metallicity technique is the efficiency of estimating metallicities for individual mainsequence stars, which are the most plentiful and representative sample of stellar populations.

Ivezić et al. (2008b) constructed photometric metallicity relations in the $u-g$ versus $g-r$ plane using SDSS filter passbands, and studied the abundance structures of the Galaxy with an accuracy of $\sim 0.2$ dex at $g<17$. This approach is similar to the traditional $U B V$ method (e.g., Carney 1979), which relies on the strong dependence of $U$-band magnitudes on metal abundance. However, the $u$-band photometry in SDSS is limited to $u \approx 22$ (99\% detection limit). This, and the greatly deteriorating errors in SDSS $u$-band magnitudes near the faint limit, restricts photometric metallicity estimates to stars at $r \lesssim 20.8$, an insufficient depth to fully explore the VOD (see Figure 37 in Jurić et al. 2008).

In this Letter, we overcome the limitations of the $u$-band photometry in SDSS by exploring less metallicity-sensitive, but better-determined, color indices in the gri passbands. ${ }^{9}$ Turnoff stars in globular clusters have $M_{r} \sim 4$, so the SDSS survey limit in $\mathrm{gri}$ (95\% completeness limit at $r=22.1$ ) allows us to probe the halo out to $\sim 20 \mathrm{kpc}$ using stars that are $\sim 2$ mag below the main-sequence turnoff $\left(\sim 0.6 M_{\odot}\right)$.

\section{METHOD}

\subsection{Photometry}

We employed SDSS photometry from DR7 (Abazajian et al. 2009). SDSS measures the brightnesses of stars with accurate astrometric positions (Pier et al. 2003), using a dedicated

\footnotetext{
9 The $z$-band data were not used due to the bright survey limit in SDSS for
} this filter. 
$2.5 \mathrm{~m}$ telescope (Gunn et al. 2006) in five broadband filters (ugriz; Stoughton et al. 2002), on six columns of CCDs (Gunn et al. 1998), under photometric conditions (Hogg et al. 2001). Photometric calibration is carried out using observations of stars in the secondary patch transfer fields (Tucker et al. 2006), based on the Smith et al. (2002) sample of standard stars.

The rms photometric precision is 0.02 mag for sources not limited by photon statistics, and the photometric calibration is accurate to $\sim 2 \%$ in the gri bands, and $\sim 3 \%$ in $u$ and $z$ (Ivezić et al. 2003, 2004; An et al. 2008). We used photometry of stellar objects (identified as STAR in the standard SDSS photometric pipelines; Lupton et al. 2002) that were detected (at the $5 \sigma$ level) in all of the gri passbands. The observed magnitudes are corrected for extinction adopting reddening values in the Schlegel et al. (1998) dust maps and the extinction coefficients given by An et al. (2009).

\subsection{Photometric Metallicity}

The isochrones in An et al. (2009) were used to determine photometric metallicity $\left([\mathrm{Fe} / \mathrm{H}]_{\text {phot }}\right)$ estimates based on color-color relations for main-sequence stars. In the following analysis, we adopted the same $\alpha$-element enhancement scheme as in An et al. (2009), motivated by the observed behavior of these elements among field dwarfs and cluster stars from spectroscopic studies (e.g., Venn et al. 2004; Kirby et al. 2008a): $[\alpha / \mathrm{Fe}]=+0.4$ at $[\mathrm{Fe} / \mathrm{H}]=-3.0$, $[\alpha / \mathrm{Fe}]=+0.3$ at $[\mathrm{Fe} / \mathrm{H}]=-2.0,-1.5,-1.0,[\alpha / \mathrm{Fe}]=$ +0.2 at $[\mathrm{Fe} / \mathrm{H}]=-0.5$, and $[\alpha / \mathrm{Fe}]=+0.0$ at $[\mathrm{Fe} / \mathrm{H}]=$ $-0.3,-0.2,-0.1,+0.0,+0.1,+0.2,+0.4$. A linear interpolation was used in this metallicity grid to obtain isochrones at intermediate $[\mathrm{Fe} / \mathrm{H}]$ values. We adopted an age of $12.6 \mathrm{Gyr}$ for $[\mathrm{Fe} / \mathrm{H}] \leqslant-1$, and $4.6 \mathrm{Gyr}$ at $[\mathrm{Fe} / \mathrm{H}] \geqslant 0$, and a linear interpolation between these two values. To expedite the computational process, we derived fifth-order polynomials to describe the color-magnitude relations, with intervals in abundance of $\Delta[\mathrm{Fe} / \mathrm{H}]=0.01 \mathrm{dex}$.

To reliably estimate a photometric metal abundance, it is necessary to use stellar models that match cluster main sequences and give internally consistent distances from multiple colormagnitude diagrams (CMDs). However, despite improvements in theoretical models, there still exist small but significant mismatches between calculated isochrone colors and the best photometry in well-studied open clusters (Pinsonneault et al. 2004; An et al. 2007a, 2007b). Therefore, we applied empirical corrections on theoretical color $-T_{\text {eff }}$ relations for $[\mathrm{Fe} / \mathrm{H}] \geqslant-0.8$ to match the observed main sequence of the solar-metallicity cluster M67 (An et al. 2009). Constant correction factors were adopted at $[\mathrm{Fe} / \mathrm{H}] \geqslant 0$, and a linear ramp was used between $[\mathrm{Fe} / \mathrm{H}]=-0.8$ and $[\mathrm{Fe} / \mathrm{H}]=0$, so that the correction becomes zero at $[\mathrm{Fe} / \mathrm{H}]=-0.8$ and below, as the models are in good agreement with the data for globular clusters (An et al. 2009).

A photometric metallicity (An et al. 2007b) was computed for each star by requiring distances from main-sequence fitting to be the same from two different CMDs, with $g-r$ and $g-i$ as color indices and $r$ as a luminosity index (Figure 1). We searched the entire $[\mathrm{Fe} / \mathrm{H}]$ grid from -3.0 to +0.4 to find $[\mathrm{Fe} / \mathrm{H}]$ with a minimum $\chi^{2}$ value, defined as

$$
\chi^{2}=\frac{\left(\mu_{g-r}-\bar{\mu}\right)^{2}}{\sigma_{\mu_{g-r}}^{2}}+\frac{\left(\mu_{g-i}-\bar{\mu}\right)^{2}}{\sigma_{\mu_{g-i}}^{2}},
$$

for each star. Here, $\mu$ and $\sigma_{\mu}$ are the distance modulus and its error for each CMD, respectively. The quantity $\bar{\mu}$ is a weighted

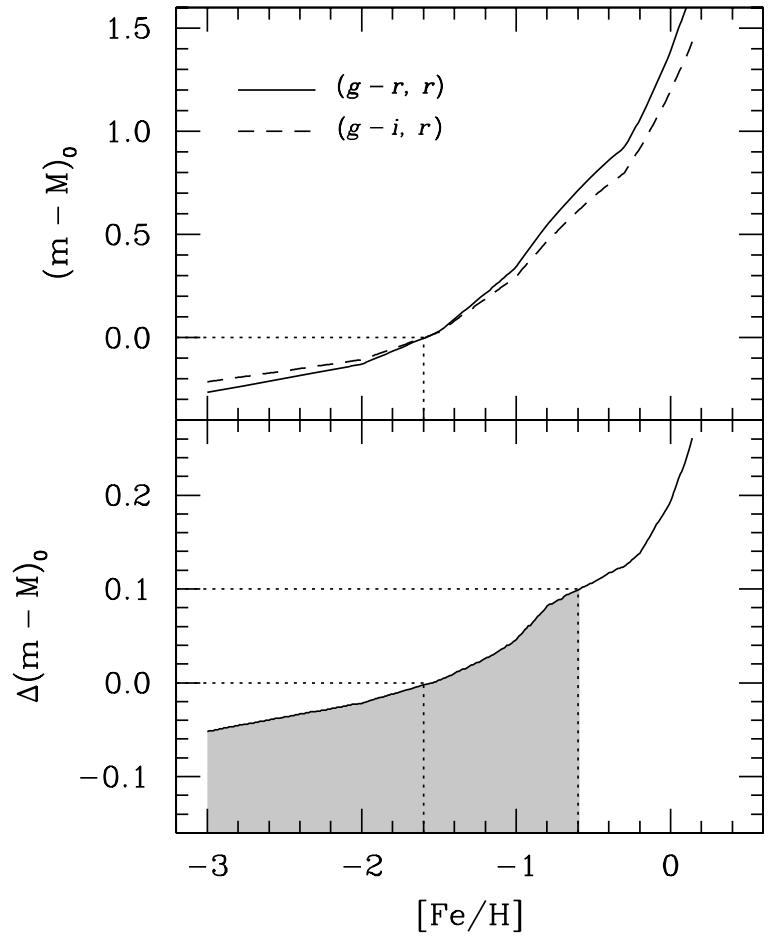

Figure 1. Illustration of the photometric metallicity estimation technique. Top: distance moduli from two CMDs as a function of a metallicity. For this example, $[\mathrm{Fe} / \mathrm{H}]=-1.6$ was assumed for the true metallicity of a star. Bottom: difference in distance modulus from the two CMDs. A photometric metallicity is defined as the $[\mathrm{Fe} / \mathrm{H}]$ that results in the same distance modulus from the two CMDs. The gray region shows the error bound for a $\sim 2 \%$ photometric color error. Note that only the upper $[\mathrm{Fe} / \mathrm{H}]$ bound is defined in this example.

average distance modulus from the $(g-r, r)$ and $(g-i, r)$ CMDs. Since only three passbands are considered here, the problem is reduced to the traditional manner of determining metal abundances from a color-color diagram. In some cases, a star becomes bluer than the main-sequence turnoff as the metallicity increases, either due to a large photometric error or a younger age than our assumed values in the models. If a minimum $\chi^{2}$ was not found, $[\mathrm{Fe} / \mathrm{H}]_{\text {phot }}$ was estimated based on a mean relation between $[\mathrm{Fe} / \mathrm{H}]$ and $\Delta(m-M)_{0}$.

\subsection{Accuracy of Photometric Metallicity Determinations}

Photometric metallicity estimates become insensitive for very metal-poor stars, as illustrated in Figure 1. Therefore, $[\mathrm{Fe} / \mathrm{H}]_{\text {phot }}$ could be biased due to systematic effects such as photometric errors, unresolved binary stars, and dust extinction, which alter the differential color estimates at a small level. We tested our photometric metallicity estimates using field stars with wellmeasured spectroscopic estimates, as discussed below, and adjusted $[\mathrm{Fe} / \mathrm{H}]_{\text {phot }}$ to correct for the bias.

Figure 2 shows the comparison with low-resolution $(R \sim$ 2000) spectroscopic measurements for field dwarfs (Carollo et al. 2007; Yanny et al. 2009) from the most recent version (Data Release planned in 2010 December) of the SEGUE Stellar Parameter Pipeline (SSPP; Lee et al. 2008a, 2008b). This version of SSPP partially solves the under- and overestimation of metallicity at higher and lower ends of $[\mathrm{Fe} / \mathrm{H}]$ that the earlier version (DR7) showed. Comparisons are shown for 46,983 stars, after applying cuts at $\mathrm{S} / \mathrm{N}>20$ and $\log g \geqslant 4.2$ to select dwarfs with good spectroscopic abundance measurements. We further restricted the sample to those with $\left|1.4(g-r)_{0}-(g-i)_{0}\right| \leqslant 0.1$. Open points show a median metallicity in $[\mathrm{Fe} / \mathrm{H}]=0.2 \mathrm{dex}$ 


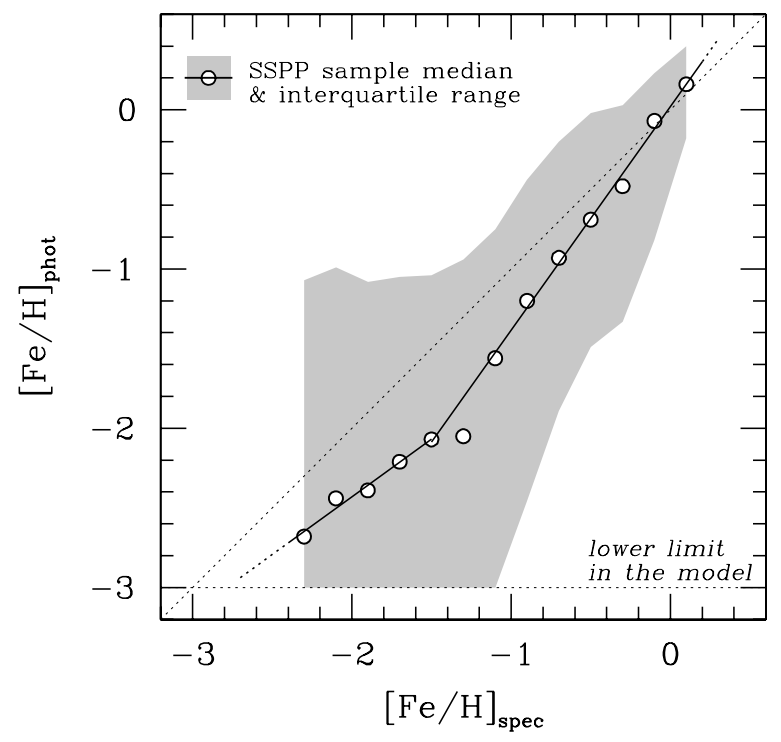

Figure 2. Comparison between spectroscopic and photometric estimates of $[\mathrm{Fe} / \mathrm{H}]$. Open circles are median $[\mathrm{Fe} / \mathrm{H}]_{\text {phot }}$ for the SSPP field star sample with available spectroscopic metallicities; the gray region represents interquartile ranges. Solid lines are piecewise linear fits to the open circles.

bins; the gray region represents interquartile ranges. Because the metallicity sensitivity essentially disappears below the lower limit of our metallicity grid, a significant number of stars are found at $[\mathrm{Fe} / \mathrm{H}]_{\text {phot }}=-3.0$.

The large uncertainty in $[\mathrm{Fe} / \mathrm{H}]_{\text {phot }}$ is mainly due to photometric errors. We performed artificial star tests by generating stars from the isochrones with Gaussian errors of 0.02 mag in $r$, $g-r$, and $g-i$. The interquartile range from the test showed a reasonable agreement with those for the SSPP sample, indicating that the observed dispersion is at least in part due to the $\sim 2 \%$ photometric error in SDSS. Despite the large uncertainty in $[\mathrm{Fe} / \mathrm{H}]_{\text {phot }}$ for individual stars, a meaningful constraint on a median $[\mathrm{Fe} / \mathrm{H}]_{\text {phot }}$ can be made by applying the technique to a large number of stars $(N \sim 500$, see below $)$.

For the SSPP sample in Figure 2, we estimated the error in the median of $\lesssim 0.1 \mathrm{dex}$ at $[\mathrm{Fe} / \mathrm{H}]_{\text {spec }}>-2$ in each $[\mathrm{Fe} / \mathrm{H}]$ bin; this was done by computing the median absolute deviation $(\mathrm{MAD})^{10}$ from stars with $[\mathrm{Fe} / \mathrm{H}]_{\text {phot }}$ above the median, divided by $\sqrt{N}$ (although $[\mathrm{Fe} / \mathrm{H}]_{\text {phot }}$ does not strictly follow a normal distribution). We explored the effects of differing stellar ages $(\sigma=20 \%),[\alpha / \mathrm{Fe}] \operatorname{ratios}(\sigma \approx 0.1 \mathrm{dex}$ at $[\mathrm{Fe} / \mathrm{H}] \lesssim-1)$, and dust extinctions $(\sigma=20 \%)$, but they change $[\mathrm{Fe} / \mathrm{H}]_{\text {phot }}$ by $\lesssim 0.1$ dex.

As shown in Figure 2, our photometric solution underestimates $[\mathrm{Fe} / \mathrm{H}]$ by as much as $\Delta[\mathrm{Fe} / \mathrm{H}] \sim 0.5$ dex for $[\mathrm{Fe} / \mathrm{H}] \lesssim-1$ with respect to the SSPP results. One reason for this offset is likely due to the presence of unresolved binary populations in the sample, which will have a portion of their light from a cooler secondary (An et al. 2007b). To evaluate the effect of binaries, we performed artificial star tests with binaries generated from a flat mass function for the secondaries, and found that the $\Delta[\mathrm{Fe} / \mathrm{H}] \sim 0.5$ dex offset can be explained with a $\sim 40 \%$ binary fraction. On the other hand, the difference could also be induced by a systematic color offset in the isochrones. Although we found a good agreement of the models with globular cluster data within the errors (An et al. 2009), a small color offset $(\lesssim 0.01 \mathrm{mag})$ in the models can still change

\footnotetext{
${ }^{10} \mathrm{MAD} \equiv 1.483 \operatorname{median}\left(\left|x_{i}-\operatorname{median}\left(x_{i}\right)\right|\right)$
}

the photometric metallicity estimate by $\Delta[\mathrm{Fe} / \mathrm{H}] \sim 0.5$ dex at the low-metallicity end. Although possible, the low-resolution spectroscopic values are less likely the source of the problem, given the extensive tests that have been applied in their validation (see Lee et al. 2008a, 2008b; Allende Prieto et al. 2008). Nevertheless, the relative metallicity comparison would be robust for stellar populations with the same binary fraction, even if there is an offset with the SSPP.

In the following analysis, we adjusted our $[\mathrm{Fe} / \mathrm{H}]_{\text {phot }}$ to be on the same scale as the SSPP results by deriving a piecewise linear fit to the median $[\mathrm{Fe} / \mathrm{H}]_{\text {phot }}$ as a function of metallicity (solid lines in Figure 2). This adjustment implicitly assumes the same fraction of unresolved binaries applies to both the SSPP and the field halo samples, if binaries are solely responsible for the difference between the $[\mathrm{Fe} / \mathrm{H}]_{\text {phot }}$ and the SSPP results. No statistically significant trend was found in the systematic difference between $[\mathrm{Fe} / \mathrm{H}]_{\text {phot }}$ and $[\mathrm{Fe} / \mathrm{H}]_{\text {spec }}$ for various sets of the binned data with different magnitudes and color ranges.

\section{RESULTS}

Figure 3 shows the distribution of stellar number density (left) and photometric metallicity (right) for 740,658 stars detected in SDSS. The maps are Lambert equal-area projections of the northern Galactic hemisphere, and the distributions are projected as seen from the Sun (i.e., a view from the inside of the Galaxy). The north Galactic pole is located at the center, and the direction of the Galactic center is toward the bottom in each panel. Each pixel has an area of $12.96 \mathrm{deg}^{2}$. To avoid any possible bias, we restricted our sample to stars with $0.3 \leqslant(g-r)_{0} \leqslant 0.4$ and the same color-color cut as those for the SSPP comparison sample. We used stars with $15.0 \leqslant(m-M)_{0} \leqslant 16.5(10 \leqslant d \leqslant 20 \mathrm{kpc})$. Although a distance modulus was derived from the $\chi^{2}$ minimization for each star, we used a $12.6 \mathrm{Gyr}$ old model with $[\mathrm{Fe} / \mathrm{H}]=-1.6$ for all of the stars to bracket the distance range in the sample. This was because the uncertainty in $[\mathrm{Fe} / \mathrm{H}]_{\text {phot }}$ for individual stars is large enough that the strong correlation between $[\mathrm{Fe} / \mathrm{H}]_{\text {phot }}$ and distance could lead to a biased result.

The feature at $(l, b) \sim\left(300^{\circ}, 70^{\circ}\right)$ is the VOD (Juric et al. $2008)$, and the long strip that crosses the sky from $(l, b) \sim$ $\left(210^{\circ}, 30^{\circ}\right)$ to the VOD is the leading tidal tail of Sgr. The feature at $(l, b) \sim\left(40^{\circ}, 40^{\circ}\right)$ is the Hercules-Aquila cloud, another large-area overdensity of halo stars discovered from SDSS (Belokurov et al. 2007).

The median metallicity of stars in the VOD area at $270^{\circ} \leqslant$ $l \leqslant 330^{\circ}$ and $60^{\circ} \leqslant b \leqslant 70^{\circ}$ is $[\mathrm{Fe} / \mathrm{H}]=-2.0 \pm 0.1$ from the metallicity map in Figure 3, where the error is from a pixel-topixel dispersion. The photometric zero points in SDSS vary at the $\sim 2 \%$ level over a $\sim 10^{\prime}$ scale along the scan line (An et al. 2008). They are also known to vary at the same $\sim 2 \%$ level over a larger angular scale along the stripe (a 2.5 wide SDSS stripe typically runs from the first to the third Galactic quadrant), and from one strip to the other (Ivezić et al. 2003, 2004). Our hope is that these components are properly averaged out over a large area in the sky, such as the solid angle covered by the VOD.

Field halo stars located at the mirrored position $\left(30^{\circ} \leqslant\right.$ $\left.l \leqslant 90^{\circ}, 60^{\circ} \leqslant b \leqslant 70^{\circ}\right)$ exhibit $[\mathrm{Fe} / \mathrm{H}]=-1.9 \pm 0.1$. Although half of the stars in the direction toward Virgo are likely associated with a progenitor dwarf galaxy or a tidal stream, the median $[\mathrm{Fe} / \mathrm{H}]$ value essentially remains unchanged; it is the same as that for the field halo stars within the precision of the technique. 

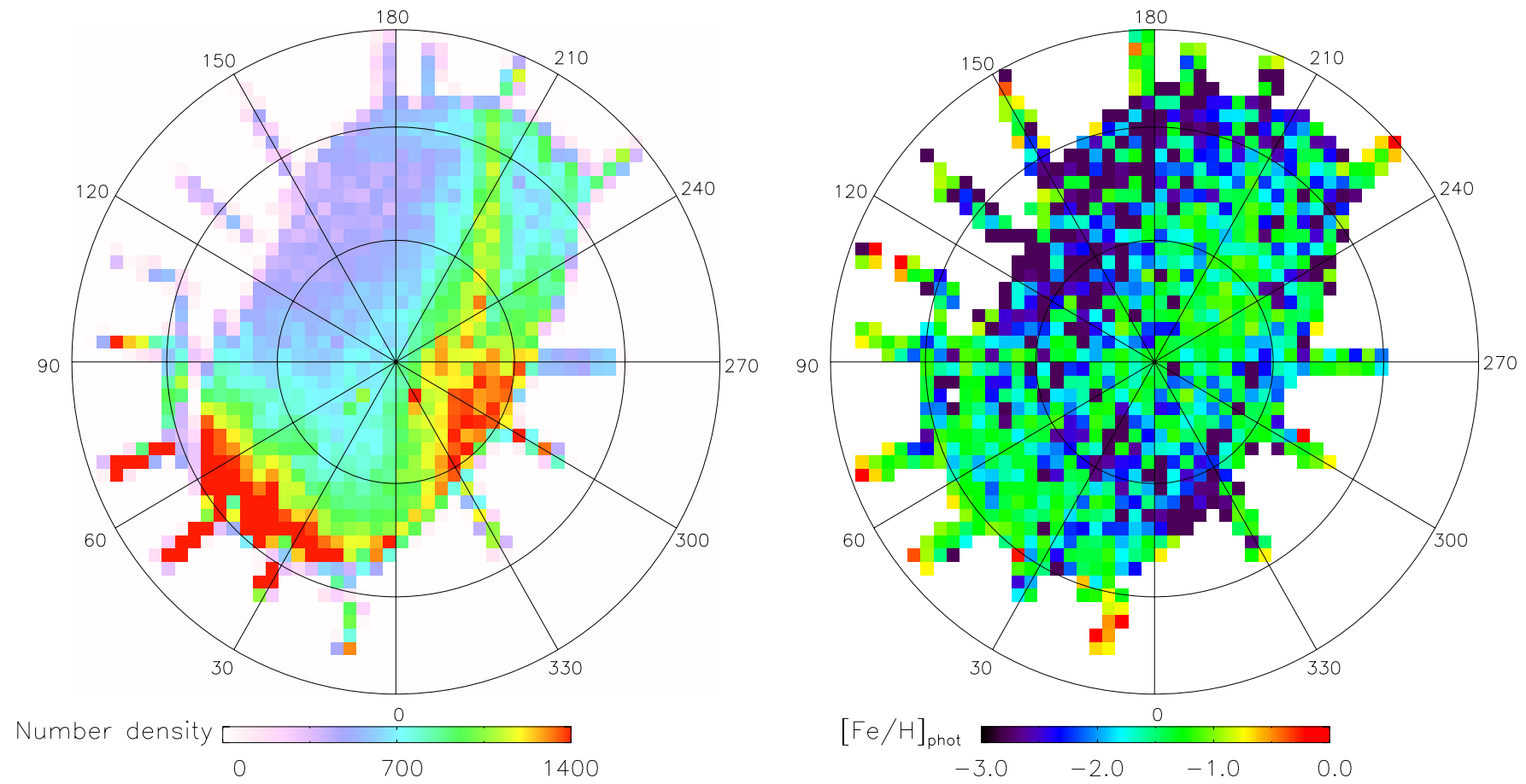

Figure 3. Left: number density of stars at distances from $\sim 10 \mathrm{kpc}$ to $\sim 20 \mathrm{kpc}$ from the Sun in the Lambert projection of the Galactic coordinates. The North Galactic pole is at the center, and the Galactic Center is to the bottom. Concentric circles represent $b=0^{\circ}, 30^{\circ}$, and $60^{\circ}$, respectively. The VOD is the feature seen at $(l, b) \sim\left(300^{\circ}, 70^{\circ}\right)$. Right: median metallicity of the same stars as in the left panel. The median occupancy of each pixel is 544 for both maps.

It is perhaps of interest that the field-star metallicities are as low as they appear to be, as Carollo et al. (2007) have argued that the peak metallicity of the outer-halo population is $[\mathrm{Fe} / \mathrm{H}]=-2.2$, and that this component is expected to dominate over the more metal-rich inner-halo population (with a peak metallicity at $[\mathrm{Fe} / \mathrm{H}]=-1.6$ ) at Galactocentric distances greater than $15-20 \mathrm{kpc}$. Note that metallicity estimates at the second Galactic quadrant are even lower than $[\mathrm{Fe} / \mathrm{H}] \approx-2$.

In Table 1, we list the sources of several systematic errors and their contributions to errors in $[\mathrm{Fe} / \mathrm{H}]_{\text {phot }}$ for the VOD. The effects of the age, extinction, and $[\alpha / \mathrm{Fe}]$ were tested by constructing similar $[\mathrm{Fe} / \mathrm{H}]_{\text {phot }}$ maps to Figure 3 with different parameters in the models. Our photometric technique is valid only for main-sequence stars, but giants constitute approximately $10 \%$ of the stars in the sample (see An et al. 2008). Since photometry alone cannot be used to adequately distinguish giants from dwarfs, we estimated a bias due to the presence of giants using photometry for a sample of nearby globular clusters (An et al. 2008). In Table 1, we also list the error from an alternative approach where we used a median difference in distance modulus in each pixel of Figure 3 to determine $[\mathrm{Fe} / \mathrm{H}]_{\text {phot }}$. The total systematic error is the quadrature sum of all of the error contributions. The relative metallicity comparison is more robust, if stellar populations in the field halo and the VOD have the same age and $[\alpha / \mathrm{Fe}]$ distributions: the difference in $[\mathrm{Fe} / \mathrm{H}]_{\text {phot }}$ remains within $\Delta[\mathrm{Fe} / \mathrm{H}]_{\text {phot }} \lesssim 0.2$.

\section{DISCUSSION}

This initial application of the photometric metallicity technique demonstrated that there is no metallicity difference between the field halo stars and those in the VOD within the precision of the method. Our estimate can be compared with previous measurements for a handful of RR Lyrae variables that are likely associated with the VOD (Duffau et al. 2006; Vivas et al. 2008;
Table 1

Error Budget in $[\mathrm{Fe} / \mathrm{H}]_{\text {phot }}$ for the VOD

\begin{tabular}{lcc}
\hline \hline \multicolumn{1}{c}{ Source of Error } & $\Delta$ Quantity & $\Delta[\mathrm{Fe} / \mathrm{H}]_{\text {phot }}$ \\
\hline Internal & $\ldots$ & \pm 0.1 \\
Age & $\pm 20 \%$ & \pm 0.3 \\
Dust extinction & $\pm 20 \%$ & \pm 0.1 \\
{$[\alpha / \mathrm{Fe}]$} & $\pm 0.1 \mathrm{dex}$ & \pm 0.3 \\
Contamination by giants & $\ldots$ & -0.1 \\
Alternative approach & $\ldots$ & -0.1 \\
Total (systematic) & $\ldots$ & \pm 0.5 \\
\hline
\end{tabular}

Note. These estimates are slightly different from those for the SSPP sample (Section 2.3) due to different color ranges used.

Prior et al. 2009). These estimates range from $[\mathrm{Fe} / \mathrm{H}]=-1.55$ to -1.95 , based on the pseudo-equivalent width of the Ca II $\mathrm{K}$ line. From principal axes on the $u-g$ versus $g-r$ diagram, Jurić et al. (2008) argued that the VOD metallicity is lower than that of thick-disk stars and similar to that of halo stars.

It is tempting to place the VOD in the observed trend of the luminosity-metallicity relation among surviving dwarf galaxies in the Local Group (e.g., Grebel et al. 2003; Muñoz et al. 2006; Kirby et al. 2008b). If we take our median $[\mathrm{Fe} / \mathrm{H}]$ from Figure 3 at $M_{V} \sim 10$ (Jurić et al. 2008; Prior et al. 2009), the VOD follows this trend, supporting the idea that $[\mathrm{Fe} / \mathrm{H}]$ can serve as a luminosity indicator for an accreting dwarf galaxy, in the process of building up the stellar halos of large spiral galaxies like the Milky Way (e.g., Johnston et al. 2008). However, this should be taken with caution, as previous studies often report average abundances for dwarf galaxies rather than median values.

Future imaging surveys, such as the Large Synoptic Survey Telescope (LSST; Ivezić et al. 2008a) will use similar photometric bandpasses as those in SDSS, providing even deeper (and far more accurate) photometric data than SDSS over a larger fraction of the sky. Our photometric metallicity 
method will be useful to exploit these databases for understanding the chemical evolution of progenitor dwarf galaxies that are identified, as well as for the bulk populations of field stars.

We thank James Bullock, Željko Ivezić, Heather Morrison, and Katie Schlesinger for useful discussions. T.C.B. and Y.S.L. acknowledge partial funding of this work from grant PHY 0822648: Physics Frontiers Center/Joint Institute for Nuclear Astrophysics (JINA), awarded by the U.S. National Science Foundation. Funding for the SDSS and SDSS-II has been provided by the Alfred P. Sloan Foundation, the Participating Institutions, the National Science Foundation, the U.S. Department of Energy, the National Aeronautics and Space Administration, the Japanese Monbukagakusho, the Max Planck Society, and the Higher Education Funding Council for England. The SDSS Web site is http://www.sdss.org/.

The SDSS is managed by the Astrophysical Research Consortium for the Participating Institutions. The Participating Institutions are the American Museum of Natural History, Astrophysical Institute Potsdam, University of Basel, University of Cambridge, Case Western Reserve University, University of Chicago, Drexel University, Fermilab, the Institute for Advanced Study, the Japan Participation Group, Johns Hopkins University, the Joint Institute for Nuclear Astrophysics, the Kavli Institute for Particle Astrophysics and Cosmology, the Korean Scientist Group, the Chinese Academy of Sciences (LAMOST), Los Alamos National Laboratory, the Max-Planck Institute for Astronomy (MPIA), the Max-Planck Institute for Astrophysics (MPA), New Mexico State University, Ohio State University, University of Pittsburgh, University of Portsmouth, Princeton University, the United States Naval Observatory, and the University of Washington.

\section{REFERENCES}

Abazajian, K., et al. 2003, AJ, 126, 2081

Abazajian, K., et al. 2004, AJ, 128, 502

Abazajian, K., et al. 2005, AJ, 129, 1755

Abazajian, K. N., et al. 2009, ApJS, 182, 543

Adelman-McCarthy, J. K., et al. 2006, ApJS, 162, 38

Adelman-McCarthy, J. K., et al. 2007, ApJS, 172, 634

Adelman-McCarthy, J. K., et al. 2008, ApJS, 175, 297

Allende Prieto, C., et al. 2008, AJ, 136, 2070

An, D., Terndrup, D. M., \& Pinsonneault, M. H. 2007a, ApJ, 671, 1640
An, D., Terndrup, D. M., Pinsonneault, M. H., Paulson, D. B., Hanson, R. B. \& Stauffer, J. R. 2007b, ApJ, 655, 233

An, D., et al. 2008, ApJS, 179, 326

An, D., et al. 2009, ApJ, 700, 523

Belokurov, V., et al. 2007, ApJ, 657, L89

Bullock, J. S., \& Johnston, K. V. 2005, ApJ, 635, 931

Carney, B. W. 1979, ApJ, 233, 211

Carollo, D., et al. 2007, Nature, 450, 1020

Duffau, S., Zinn, R., Vivas, A. K., Carraro, G., Méndez, R. A., Winnick, R., \& Gallart, C. 2006, ApJ, 636, L97

Eggen, O. J., Lynden-Bell, D., \& Sandage, A. R. 1962, ApJ, 136, 748

Grebel, E. K., Gallagher, J. S., III, \& Harbeck, D. 2003, AJ, 125, 1926

Gunn, J. E., et al. 1998, AJ, 116, 3040

Gunn, J. E., et al. 2006, AJ, 131, 2332

Hogg, D. W., Finkbeiner, D. P., Schlegel, D. J., \& Gunn, J. E. 2001, AJ, 122 2129

Ivezić, Z., Tyson, J. A., Allsman, R., Andrew, J., Angel, R., \& for the LSST Collaboration 2008a, arXiv:0805.2366

Ivezić, Ž., et al. 2003, Mem. Soc. Astron. Ital., 74, 978

Ivezić, Ž., et al. 2004, Astron. Nachr., 325, 583

Ivezić, Ž., et al. 2008b, ApJ, 684, 287

Johnston, K. V., Bullock, J. S., Sharma, S., Font, A., Robertson, B. E., \& Leitner, S. N. 2008, ApJ, 689, 936

Jurić, M., et al. 2008, ApJ, 673, 864

Kirby, E. N., Guhathakurta, P., \& Sneden, C. 2008a, ApJ, 682, 1217

Kirby, E. N., Simon, J. D., Geha, M., Guhathakurta, P., \& Frebel, A. 2008b, ApJ, $685, \mathrm{~L} 43$

Lee, Y. S., et al. 2008a, AJ, 136, 2022

Lee, Y. S., et al. 2008b, AJ, 136, 2050

Lupton, R. H., Ivezic, Z., Gunn, J. E., Knapp, G., Strauss, M. A., \& Yasuda, N. 2002, Proc. SPIE, 4836, 350

Muñoz, R. R., Carlin, J. L., Frinchaboy, P. M., Nidever, D. L., Majewski, S. R., \& Patterson, R. J. 2006, ApJ, 650, L51

Newberg, H. J., Yanny, B., Cole, N., Beers, T. C., Re Fiorentin, P., Schneider, D. P., \& Wilhelm, R. 2007, ApJ, 668, 221

Newberg, H. J., et al. 2002, ApJ, 569, 245

Pier, J. R., Munn, J. A., Hindsley, R. B., Hennessy, G. S., Kent, S. M., Lupton, R. H., \& Ivezić, Ž. 2003, AJ, 125, 1559

Pinsonneault, M. H., Terndrup, D. M., Hanson, R. B., \& Stauffer, J. R. 2004, ApJ, 600, 946

Prior, S. L., Da Costa, G. S., Keller, S. C., \& Murphy, S. J. 2009, ApJ, 691, 306 Schlegel, D. J., Finkbeiner, D. P., \& Davis, M. 1998, ApJ, 500, 525

Searle, L., \& Zinn, R. 1978, ApJ, 225, 357

Smith, J. A., et al. 2002, AJ, 123, 2121

Stoughton, C., et al. 2002, AJ, 123, 485

Tucker, D. L., et al. 2006, Astron. Nachr., 327, 821

Venn, K. A., Irwin, M., Shetrone, M. D., Tout, C. A., Hill, V., \& Tolstoy, E. 2004, AJ, 128, 1177

Vivas, A. K., Jaffé, Y. L., Zinn, R., Winnick, R., Duffau, S., \& Mateu, C. 2008, AJ, 136, 1645

Vivas, A. K., et al. 2001, ApJ, 554, L33

Yanny, B., et al. 2009, AJ, 137, 4377

York, D. G., et al. 2000, AJ, 120, 1579 